\title{
Bounds on Scattering Poles in One Dimension
}

\author{
Michael Hitrik \\ Department of Mathematics, University of Lund, Box 118 S-221 00 Lund, Sweden \\ E-mail: mike@maths.lth.se
}

\begin{abstract}
For the class of super-exponentially decaying potentials on the real line sharp upper bounds on the counting function of the poles in discs are derived and the density of the poles in strips is estimated. In the case of nonnegative potentials, explicit estimates for the width of a pole-free strip are obtained.
\end{abstract}

\section{Introduction}

The purpose of this paper is to establish some estimates on the scattering poles for the class of exponentially and super-exponentially decaying potentials on the real line. In particular, we derive sharp upper bounds on the counting function for the poles of super-exponentially decaying potentials and estimate the density of the poles in strips. In the case of nonnegative potentials we give explicit estimates for the size of a polefree strip. These results are obtained using the representation of the scattering matrix, given by Melin [14]. Notice that this representation has also been used by Zworski [17] in his study of the distribution of scattering poles in the case of compactly supported potentials.

We also present an alternative approach to the study of the location of the poles, obtained by rewriting the Schrödinger equation as a system of Riccati equations.

The existence of pole-free regions and estimates of their size is one of the main problems in the theory of resonances. This problem has been investigated extensively 
in the semi-classical setting and in the framework of the Lax-Phillips theory. Also, the problem of estimating the number of scattering poles in various subdomains of the complex plane has been much studied in the recent years. The survey paper [18] contains an overview of this work as well as an extensive bibliography.

The results of this paper are concerned with the class of (super-)exponentially decaying potentials, and when deriving bounds on the scattering poles, we shall always indicate explicitly their dependence on the potential.

The organization of the paper is as follows. In the beginning of Section 2 we recall the intertwining operators and the representation of the scattering matrix, as given in [14]. We also discuss some continuity properties of the scattering matrix as a function of the potential. The basic estimates for the study of scattering poles are then derived in Theorem 2.4 and Theorem 2.5, and the density of the poles for superexponentially decaying potentials in discs is estimated. In the beginning of Section 3 we estimate the size of a pole-free strip for compactly supported nonnegative potentials, and afterwards this estimate is generalized to super-exponentially decaying potentials. Next we address the problem of estimating the density of poles in arbitrary strips. The obtained higher value of the density in this case compared with the case of discs reflects bounds on the location of the poles. Such bounds are finally derived using the Riccati equation approach.

\section{The scattering matrix and global upper bounds}

\subsection{The scattering data}

We begin by recalling some important results of scattering theory on the line. Our basic reference here is the paper [14].

Consider the Schrödinger equation

$$
H_{p} u=-u^{\prime \prime}+p u=k^{2} u, \quad k \in \mathbf{R},
$$

where $p$ is a real-valued measurable potential such that

$$
\int_{-\infty}^{\infty}(1+|x|)|p(x)| d x<+\infty
$$

There exist two functions $f(x, k)$ and $g(x, k)$, such that $f$ and $g$ solve $(2.1)$, and

$$
\begin{gathered}
f(x, k)=e^{i x k}+o(1), \quad x \rightarrow+\infty, \\
g(x, k)=e^{-i x k}+o(1), \quad x \rightarrow-\infty .
\end{gathered}
$$

We shall say that $f$ and $g$ are the Jost functions. For $k \in \mathbf{R} \backslash\{0\}, f(x, k)$ and $\overline{f(x, k)}=f(x,-k)$ are solutions of the same equation (2.1), but with different boundary conditions at $+\infty$, so they are linearly independent. Therefore, we can write

$$
i k g(x, k)=a(k) f(x,-k)+b(k) f(x, k),
$$


where $a(k)$ and $b(k)$ are uniquely determined. One finds that $\overline{a(k)}=a(-k), \overline{b(k)}=$ $b(-k)$, and that

$$
k^{2}+|b(k)|^{2}=|a(k)|^{2} .
$$

A combination of (2.3) with its complex conjugate then shows that

$$
i k f(x, k)=a(k) g(x,-k)+b(-k) g(x, k) .
$$

We shall now introduce the elements of the scattering matrix of $p$ when $0 \neq k \in \mathbf{R}$. When doing it we notice that, since $a(k) \neq 0$ by (2.4), it follows that the functions $f$ and $g$ form a basis of solutions of (2.1). Moreover, they extend to analytic functions of $k$ in the upper half-plane, continuous in the closure of that set. Their complex conjugates $\overline{f(x, k)}=f(x,-k)$ and $\overline{g(x, k)}=g(x,-k)$ have natural analytic extensions to the lower half-plane instead. The equations (2.3) and (2.5) may now be rewritten in the form

$$
\begin{gathered}
f(x,-k)=\frac{i k}{a(k)} g(x, k)-\frac{b(k)}{a(k)} f(x, k), \\
g(x,-k)=-\frac{b(-k)}{a(k)} g(x, k)+\frac{i k}{a(k)} f(x, k),
\end{gathered}
$$

where we have expressed the solutions of (2.1) with analytic extensions to $\mathbf{C}_{-}$as linear combinations of those with analytic extensions to $\mathbf{C}_{+}$. Since $f(x, k)$ was normalized by boundary conditions at $+\infty$, we shall call $r(k)=b(k) / a(k)$ the right reflection coefficient. For similar reasons, $b(-k) / a(k)$ is called the left reflection coefficient, and the function $t(k)=i k / a(k)$ is the transmission coefficient. We notice that the matrix appearing in (2.6) is unitary. This is the scattering matrix, after the off-diagonal elements have been multiplied by -1 .

The assertions about analyticity in $k \in \mathbf{C}_{+}$of the functions $f$ and $g$ are consequences of their integral representations in terms of the intertwining operators between $H_{p}$ and $H_{0}$, which we proceed to discuss following [14]. Associated to $p$, there are two operators $A_{+}=I+R_{+}$and $A_{-}=I+R_{-}$, with $H_{p} A_{ \pm}=A_{ \pm} H_{0}$, such that $\pm(y-x) \geq 0$ in the support of $A_{ \pm}$. (Here and in what follows we identify operators with their distribution kernels.) The functions $R_{ \pm}$are continuous up to the boundary in the sets $\pm(y-x)>0$, and

$$
\left\|R_{ \pm}(x, \cdot)\right\|_{L^{1}}=\int\left|R_{ \pm}(x, y)\right| d y<\infty
$$

for any $x$. Moreover, $\left\|R_{ \pm}(x, \cdot)\right\|_{L^{1}} \rightarrow 0$ as $x \rightarrow \pm \infty$. It follows then that $f(x,-k)$ (resp. $g(x, k))$ is the Fourier transform of $A_{+}(x, y)$ (resp. $\left.A_{-}(x, y)\right)$ with respect to the second variable, so that

$$
f(x, k)=e^{i x k}+\int_{x}^{\infty} R_{+}(x, y) e^{i y k} d y
$$


and

$$
g(x, k)=e^{-i x k}+\int_{-\infty}^{x} R_{-}(x, y) e^{-i y k} d y
$$

where $k \in \mathbf{R}$.

Apart from functions that are continuous on the whole of $\mathbf{R}^{2}$, we have

$$
R_{ \pm}(x, y) \equiv R_{ \pm, 0}(x, y)
$$

where

$$
R_{+, 0}(x, y)=\left(\frac{1}{2}\right) \theta_{+}(y-x) \int_{(x+y) / 2}^{\infty} p(t) d t
$$

and

$$
\begin{gathered}
R_{-, 0}(x, y)=\left(\frac{1}{2}\right) \theta_{+}(x-y) \int_{-\infty}^{(x+y) / 2} p(t) d t \\
\theta_{+}(t)=1 \quad \text { when } t \geq 0 \quad \text { and } 0 \text { otherwise. }
\end{gathered}
$$

These are the leading terms in $R_{ \pm}$, and one has that the $R_{ \pm}$satisfy the equations

$$
R_{ \pm}=R_{ \pm, 0}+L_{p, \pm} R_{ \pm}
$$

where $L_{p}=L_{p,-}$ is given by

$$
\begin{aligned}
& L_{p} T(x, y)=\iint E\left(x-x^{\prime}, y-y^{\prime}\right) p\left(x^{\prime}\right) T\left(x^{\prime}, y^{\prime}\right) d x^{\prime} d y^{\prime} \\
& E(x, y)=\frac{1}{2} \quad \text { when } x>0,|y|<|x| \text { and } 0 \text { otherwise. }
\end{aligned}
$$

There is a similar expression for $L_{p,+}(x, y)$. In what follows we shall write $R_{-, 0}(x, y)=$ $R_{0}(x, y), R_{-}(x, y)=R(x, y)$, when no confusion seems possible.

In order to describe the growth properties of $R(x, y)$, and, in particular, to sharpen (2.7), we introduce the nondecreasing functions

$$
u(x)=\int_{-\infty}^{x}|p(t)| d t, \quad v(x)=\int_{-\infty}^{x} u(t) d t
$$

The solution $R$ of (2.10) is obtained by inverting the operator $I-L_{p}$;

$$
R=\sum_{k=0}^{\infty} L_{p}^{k} R_{0}
$$

The following estimate

$$
\left|L_{p}^{k} R_{0}(x, y)\right| \leq \frac{1}{2} u\left(\frac{x+y}{2}\right) \frac{(v(x)-v((x+y) / 2))^{k}}{k !}, \quad x \geq y,
$$


is true, see [13]. Therefore,

$$
|R(x, y)| \leq \frac{1}{2} u\left(\frac{x+y}{2}\right) \exp \left(v(x)-v\left(\frac{x+y}{2}\right)\right), x \geq y,
$$

and we have

$$
\|R(x, \cdot)\|_{L^{1}}=\int_{-\infty}^{x}|R(x, y)| d y \leq e^{v(x)}-1 .
$$

Another important result ([14], Lemma 4.2) is that

$$
p(x) R(x, y) \in L^{1}\left(\mathbf{R}^{2}\right) .
$$

Notice also that if $x \geq a$ in the support of $p$, then it is immediate from (2.13) that

$$
2 a-x \leq y \leq x \text { in the support of } R(x, y) .
$$

We introduce now the following representations for the functions $a$ and $b$, given by Melin [14]: There exist temperate real-valued distributions $X$ and $Y$ such that

$$
a(k)=\hat{X}(k) \quad \text { and } \quad b(k)=\hat{Y}(k),
$$

where $X$ and $Y$ are given by the following explicit formulas

$$
\begin{gathered}
X(y)=\delta^{\prime}(y)-\left(\frac{1}{2}\right)\left(\int_{-\infty}^{+\infty} p(z) d z\right) \delta(y)-\left(\frac{1}{2}\right) \int_{-\infty}^{+\infty} p(z) R(z, z+y) d z, \\
Y(y)=\left(\frac{1}{4}\right) p\left(\frac{y}{2}\right)+\left(\frac{1}{2}\right) \int_{-\infty}^{+\infty} p(z) R(z, y-z) d z .
\end{gathered}
$$

Here the Fourier transform is normalized as in [8]. We remark that the expressions for $X$ and $Y$ in [14] were given in terms of the kernel $R_{+}(x, y)$, but using the identities $R_{+}(x, y, \check{p})=R(-x,-y, p), X_{\check{p}}=X_{p}$ and $Y_{\check{p}}=\check{Y}_{p}$, which appear in [14], formula (5.14), it is easy to see that the representations (2.17) and (2.18) are valid.

For future reference we rewrite now (2.3) in the form

$$
i k g(x, k)=\hat{X}(k) f(x,-k)+\hat{Y}(k) f(x, k) .
$$

A combination of (2.15) with (2.17) and (2.18) shows that

$$
\operatorname{chsupp}(Y) \subset \operatorname{chsupp}(p(\cdot / 2))
$$

and

$$
\operatorname{chsupp}(X) \subset[-2 d, 0],
$$

if $d$ is the diameter of the support of $p$. Furthermore,

$$
X(y)-\delta^{\prime}(y)+\left(\frac{1}{2}\right)\left(\int_{-\infty}^{+\infty} p(z) d z\right) \delta(y) \in L^{1} \cap L^{\infty}
$$


and

$$
Y(y)-\left(\frac{1}{4}\right) p\left(\frac{y}{2}\right) \in L^{1} \cap L^{\infty}
$$

It follows that $\hat{X}$ extends to an analytic function in $\operatorname{Im} k>0$, continuous up to the boundary. We also know that $\hat{X}(k)$ has finitely many zeros in $\operatorname{Im} k>0$, all of them simple and situated on the imaginary axis. Furthermore, $i \beta$ is a zero precisely when $-\beta^{2}$ is an eigenvalue of $H_{p}$.

\subsection{Dependence on the potential}

We shall now give some symmetry properties of the distributions $X$ and $Y$ with respect to the one-parameter groups $\delta_{\lambda} p(x)=\lambda^{2} p(\lambda x), \lambda>0$ and $\tau_{h} p(x)=p(x+h), h \in \mathbf{R}$. It follows from (2.9) and (2.11) that $R_{\delta_{\lambda} p}(x, y)=\lambda R_{p}(\lambda x, \lambda y)$. Therefore, the mappings

$$
p \rightarrow X_{p} \text { and } p \rightarrow Y_{p}
$$

commute with the action of the dilatation group. In other words,

$$
X_{\delta_{\lambda} p}=\delta_{\lambda} X_{p}, \quad Y_{\delta_{\lambda} p}=\delta_{\lambda} Y_{p}
$$

For the translation group we have instead,

$$
X_{\tau_{h} p}=X_{p}, \quad Y_{\tau_{h} p}=\tau_{2 h} Y_{p}
$$

Later we shall also need some continuity properties of the mappings

$$
p \rightarrow X_{p} \text { and } p \rightarrow Y_{p}
$$

which we proceed to discuss. When doing this, we let $q \geq 0$ be a measurable function such that

$$
\int_{-\infty}^{\infty}(1+|x|)|q(x)| d x<\infty
$$

and set

$$
B_{q}=\left\{p \in L^{1}(\mathbf{R}) ;|p| \leq q\right\}
$$

Definition 2.1 We say that a mapping $T: B_{q} \rightarrow W$, where $W$ is a Banach space, is weakly sequentially continuous, if for any sequence $p_{j}$ in $B_{q}$ converging to $p \in B_{q}$ weakly in the space of measures on $\mathbf{R}$ it is true that $T\left(p_{j}\right)$ converges to $T(p)$ in $W$.

Theorem 2.2 The mapping $p \rightarrow X_{p}-\delta^{\prime}$ is weakly sequentially continuous from $B_{q}$ to the Banach space of bounded measures on $\mathbf{R}$. 
Proof: Assume that $p_{j} \in B_{q}$ converges to $p \in B_{q}$ weakly in the space of measures. It follows then that $p_{j}$ is also convergent in the weak topology of $L^{1}(\mathbf{R})$, i.e.

$$
\left\langle u, p_{j}\right\rangle=\int u(x) p_{j}(x) d x \rightarrow\langle u, p\rangle=\int u(x) p(x) d x,
$$

for every $u \in L^{\infty}(\mathbf{R})$. In fact, we may find a sequence $u_{\nu} \in C_{0}$ tending to $u$ almost everywhere and boundedly. Since

$$
\sup _{j}\left|\left\langle p_{j}-p, u_{\nu}-u\right\rangle\right| \leq 2\left\langle q,\left|u_{\nu}-u\right|\right\rangle \rightarrow 0 \quad \text { as } \quad \nu \rightarrow \infty
$$

the assertion follows from the fact that $\left\langle p_{j}, u_{\nu}\right\rangle \rightarrow\left\langle p, u_{\nu}\right\rangle$ for every $\nu$. It follows in particular that

$$
P_{j}(x)=\int_{-\infty}^{x} p_{j}(y) d y
$$

converges pointwise to $P(x)=\int_{-\infty}^{x} p(y) d y$. Since it is equicontinuous and since $\left|p_{j}\right| \leq$ $q$, the convergence must be uniform on $\mathbf{R}$.

Let us consider now $R_{\infty}=R_{p}$ and $R_{j}=R_{p_{j}}$. Let $R_{j, \nu}$ be the contribution to $R_{j}$ which is homogeneous of degree $\nu \geq 1$ in $p_{j}$, see (2.11). Allowing $j$ to be $\infty$ and writing $p=p_{\infty}$, we have then

$$
R_{j, \nu+1}(x, y)=\iint E\left(x-x^{\prime}, y-y^{\prime}\right) p_{j}\left(x^{\prime}\right) R_{j, \nu}\left(x^{\prime}, y^{\prime}\right) d x^{\prime} d y^{\prime} .
$$

Let $R_{q, \nu}$ be the corresponding expression with $q$. Then

$$
\left|R_{j, \nu}\right| \leq R_{q, \nu}
$$

We shall prove now that

$$
R_{j} \rightarrow R_{p} \quad \text { in } \quad L_{\mathrm{loc}}^{\infty}\left(\mathbf{R}^{2}\right)
$$

as $j \rightarrow \infty$. Since it follows by (2.12) that $\sum_{\nu=1}^{\infty} R_{q, \nu}$ is convergent in $L_{\text {loc }}^{\infty}\left(\mathbf{R}^{2}\right)$, it suffices to prove that $R_{j, \nu}$ is convergent in $L_{\text {loc }}^{\infty}$ for every $\nu$. In view of (2.9) we have already seen that this is true when $\nu=1$, so let us assume that $\nu>1$ and that the statement has already been proved for lower values of $\nu$. We notice that the functions in (2.26) are equicontinuous, and therefore it is sufficient to show the pointwise convergence. Since $x^{\prime} \leq x$ in the support of the integrand in (2.26), and since

$$
\iint_{x^{\prime} \leq N}\left|p_{j}\left(x^{\prime}\right) R_{j, \nu}\left(x^{\prime}, y^{\prime}\right)\right| d x^{\prime} d y \leq \iint_{x^{\prime} \leq N} q\left(x^{\prime}\right) R_{q}\left(x^{\prime}, y^{\prime}\right) d x^{\prime} d y
$$

where the right-hand side tends to zero as $N \rightarrow-\infty$, we may replace the integration in (2.26) by an integration over a compact set in $x^{\prime}$ when proving our assertion. The support conditions on $E$ give us then also a bound on $y^{\prime}$ when $(x, y)$ is kept fixed. It is sufficient therefore to prove the pointwise convergence of $(2.26)$ when the integration is 
performed over a compact set. Since we know already that $R_{j, \nu}$ converges in $L_{\mathrm{loc}}^{\infty}$ and $\int p_{j}(x) u(x) d x$ is convergent when $u \in L^{\infty}$, our assertion follows.

Consider now the expression (2.17), which we write as

$$
X_{p_{j}}(y)=\delta^{\prime}(y)-\left(\frac{1}{2}\right)\left(\int_{-\infty}^{+\infty} p_{j}(z) d z\right) \delta(y)-\frac{1}{2} f_{p_{j}}(y)
$$

The coefficient in front of $\delta(y)$ converges to the corresponding one for $p$, and we want to show that $f_{p_{j}} \rightarrow f_{p}$ in $L^{1}$. Since

$$
\int\left|p_{j}(z) R_{j}(z, z+y)\right| d z \leq \int q(z) R_{q}(z, z+y) d z=W(y)
$$

where $W \in L^{1}$, it suffices to show that $f_{j}(y)=f_{p_{j}}(y)$ converges to $f_{p}$ in $L_{\text {loc }}^{1}$. Set

$$
W_{n}(y)=\int_{-n}^{n} q(z) R_{q}(z, z+y) d z .
$$

Then $\left|W_{n}\right| \leq W$ and $W_{n} \rightarrow W$ pointwise. It follows by Lebesgue's theorem that $W_{n} \rightarrow W$ in $L^{1}$ and that the norm in $L^{1}(\mathbf{R})$ of

$$
y \rightarrow \int_{|z|>n} p_{j}(z) R_{j}(z, z+y) d z
$$

converges to zero uniformly in $j$ as $n \rightarrow \infty$. It suffices therefore to prove the convergence in $L_{\text {loc }}^{1}(\mathbf{R})$ of

$$
y \rightarrow \int_{-n}^{n} p_{j}(z) R_{j}(z, z+y) d z
$$

to the corresponding integral when $R_{j}$ has been replaced by $R$, and $p_{j}$ by $p$. The proof is now complete, since we know that $R_{j} \rightarrow R$ in $L_{\text {loc }}^{\infty}$ and $\left\langle p_{j}, u\right\rangle \rightarrow\langle p, u\rangle$ when $u \in L^{\infty}$.

In the same way one can prove the following result.

Theorem 2.3 The mapping $p \rightarrow Y_{p}(y)-p(y / 2) / 4$ is weakly sequentially continuous from $B_{q}$ to $L^{1}(\mathbf{R})$.

\subsection{Proof of the main estimate}

We have already observed the important result (2.14), valid for all potentials, such that $(1+|x|) p(x) \in L^{1}$. The main estimate for the study of scattering poles is contained in the following theorem. Before stating it, we introduce the notation

$$
f_{p}(y)=\int_{-\infty}^{\infty} p(z) R_{p}(z, z+y) d z
$$

so that

$$
X_{p}(y)=\delta^{\prime}(y)-\frac{1}{2}\left(\int_{-\infty}^{\infty} p(z) d z\right) \delta(y)-\frac{1}{2} f_{p}(y)
$$


Theorem 2.4 Let $(1+|x|) e^{2 a|x|} p(x) \in L^{1}$ for some $a>0$, and set

$$
q(x)=e^{2 a|x|} p(x) .
$$

Then

$$
\int_{-\infty}^{\infty} e^{a|y|}\left|f_{p}(y)\right| d y \leq 2 e^{2\|p\|}\|q\|\left(\|q\|_{L^{1}}+\|q\|\|p\|_{L^{1}}\right)
$$

where

$$
\|p\|=\int_{-\infty}^{\infty}|x||p(x)| d x
$$

Proof: Since $\left|R_{p}\right| \leq R_{|p|}$, we may assume that $p \geq 0$. First we shall prove that

$$
L_{p}^{k} R_{0, p}(x, y) \leq e^{a(x+y)} L_{p}^{k} R_{0, q}(x, y),
$$

for all $k \geq 0$. We have

$$
R_{0, p}(x, y)=\frac{1}{2} \int_{-\infty}^{(x+y) / 2} e^{-2 a|t|} q(t) d t \leq e^{a(x+y)} R_{0, q}(x, y), \quad y \leq x,
$$

since $|t| \geq-(x+y) / 2$. Therefore we assume that $k \geq 1$, and (2.30) has been proved for lower values of $k$. We have

$$
\begin{gathered}
L_{p}^{k} R_{0, p}(x, y)=\iint E\left(x-x^{\prime}, y-y^{\prime}\right) p\left(x^{\prime}\right) L_{p}^{k-1} R_{0, p}\left(x^{\prime}, y^{\prime}\right) d x^{\prime} d y^{\prime} \\
\quad \leq \iint E\left(x-x^{\prime}, y-y^{\prime}\right) e^{a\left(x^{\prime}+y^{\prime}\right)} p\left(x^{\prime}\right) L_{p}^{k-1} R_{0, q}\left(x^{\prime}, y^{\prime}\right) d x^{\prime} d y^{\prime},
\end{gathered}
$$

and we only have to notice that

$$
x^{\prime}+y^{\prime} \leq x+y
$$

when $\left(x^{\prime}, y^{\prime}\right)$ is in the support of the integrand. This gives (2.30), and after a summation over $k$, we get

$$
R_{p}(x, y) \leq e^{a(x+y)} R_{p, q}(x, y)
$$

where

$$
R_{p, q}=\sum_{k=0}^{\infty} L_{p}^{k} R_{0, q} .
$$

We have that $y \leq 0$ in the support of $R_{p}(x, x+y)$ and it follows that

$$
e^{a|y|} \int_{-\infty}^{\infty} p(z) R_{p}(z, z+y) d z \leq \int_{-\infty}^{\infty} q(z) R_{p, q}(z, z+y) d z
$$

and then

$$
\left\|e^{a|\cdot|} f_{p}\right\|_{L^{1}} \leq \int_{-\infty}^{\infty} q(x)\left\|R_{p, q}(x, \cdot)\right\|_{L^{1}} d x
$$


Set $r(x)=\left\|R_{p, q}(x, \cdot)\right\|$. This function is bounded on any interval bounded to the right. We now come to estimate $r(x)$. Since $R_{p, q}=L_{p} R_{p, q}+R_{0, q}$, it follows that

$$
r(x)=\int_{-\infty}^{x}\left(x-x^{\prime}\right) p\left(x^{\prime}\right) r\left(x^{\prime}\right) d x^{\prime}+\int_{-\infty}^{x}\left(x-x^{\prime}\right) q\left(x^{\prime}\right) d x^{\prime} .
$$

We shall estimate $r(x)$ first for negative $x$. Set $q^{-}(x)=q(x)$ when $x<0$ and $q^{-}(x)=0$ for $x \geq 0$. Set also $s(x)=|x| p(x)$. If $x \leq 0$ we have

$$
r(x) \leq \int_{-\infty}^{x} s\left(x^{\prime}\right) r\left(x^{\prime}\right) d x^{\prime}+\left\|q^{-}\right\|
$$

This inequality may be written

$$
\varphi^{\prime}(x) \leq s(x) \varphi(x)+s(x)\left\|q^{-}\right\|,
$$

where $\varphi(x)=\int_{-\infty}^{x} s(y) r(y) d y$. If $S(x)=\int_{-\infty}^{x} s(y) d y$ it follows that

$$
\left(e^{-S} \varphi\right)^{\prime} \leq-\left\|q^{-}\right\| \frac{d}{d x} e^{-S}
$$

Hence $e^{-S(x)} \varphi(x) \leq\left(1-e^{-S(x)}\right)\left\|q^{-}\right\|$, and it follows that

$$
r(x) \leq \varphi(x)+\left\|q^{-}\right\| \leq e^{\left\|p^{-}\right\|}\left\|q^{-}\right\|, \quad x \leq 0 .
$$

Next we assume that $x \geq 0$. Let us set

$$
A=e^{\| p^{-}}\left\|q^{-}\right\|\left\|p^{-}\right\|_{L^{1}}+\|q\|_{L^{1}}
$$

and

$$
B=e^{\left\|p^{-}\right\|}\left\|p^{-}\right\|\left\|q^{-}\right\|+\left\|q^{-}\right\| .
$$

Then it is easily seen that

$$
r(x) \leq x \int_{0}^{x} p\left(x^{\prime}\right) r\left(x^{\prime}\right) d x^{\prime}+A x+B
$$

It follows if $\psi(x)=\int_{0}^{x} p(y) r(y) d y$ that

$$
\psi^{\prime}(x) \leq s(x) \psi(x)+A s(x)+B p(x) .
$$

If $h(x)=\int_{0}^{x} s(y) d y$ this may be written

$$
\left(e^{-h} \psi\right)^{\prime} \leq-A \frac{d}{d x} e^{-h}+B e^{-h} p(x) .
$$

Hence

$$
e^{-h} \psi \leq A\left(1-e^{-h}\right)+B\left\|p^{+}\right\|_{L^{1}}
$$


and

$$
\psi(x) \leq A\left(e^{h(x)}-1\right)+e^{h(x)} B\left\|p^{+}\right\|_{L^{1}} .
$$

Here $p^{+}(x)=p(x)$ for $x>0$ and 0 otherwise. It follows when $x \geq 0$ that

$$
\begin{aligned}
r(x) & \leq x \psi(x)+A x+B \leq A x e^{h}+B x e^{h}\left\|p^{+}\right\|_{L^{1}}+B \\
& \leq x e^{\left\|p^{+}\right\|}\left(A+B\left\|p^{+}\right\|_{L^{1}}\right)+B .
\end{aligned}
$$

We have

$$
\begin{aligned}
A+B\left\|p^{+}\right\|_{L^{1}} & \leq e^{\left\|p^{-}\right\|}\left\|q^{-}\right\|\|p\|_{L^{1}}+\|q\|_{L^{1}} \\
& +e^{\left\|p^{-}\right\|}\left\|p^{-}\right\|\left\|q^{-}\right\|\|p\|_{L^{1}}+\left\|q^{-}\right\|\left\|p^{+}\right\|_{L^{1}} \\
& =e^{\left\|p^{-}\right\|}\|p\|_{L^{1}}\left\|q^{-}\right\|\left(1+\left\|p^{-}\right\|\right)+\|q\|_{L^{1}}+\left\|q^{-}\right\|\left\|p^{+}\right\|_{L^{1}} \\
& \leq\|p\|_{L^{1}} e^{2\left\|p^{-}\right\|}\left\|q^{-}\right\|+\|q\|_{L^{1}}+\left\|q^{-}\right\|\|p\|_{L^{1}} \\
& \leq 2 e^{2\left\|p^{-}\right\|}\|p\|_{L^{1}}\left\|q^{-}\right\|+\|q\|_{L^{1}},
\end{aligned}
$$

and

$$
B \leq e^{\left\|p^{-}\right\|}\left\|q^{-}\right\|\left(1+\left\|p^{-}\right\|\right) \leq e^{2\left\|p^{-}\right\|}\left\|q^{-}\right\| .
$$

Hence it follows that for $x \geq 0$

$$
r(x) \leq x e^{2\|p\|}\left(2\|p\|_{L^{1}}\left\|q^{-}\right\|+\|q\|_{L^{1}}\right)+e^{2\|p\|}\left\|q^{-}\right\| .
$$

Combining it with (2.32) we obtain

$$
\begin{aligned}
\int_{-\infty}^{\infty} q(x) r(x) d x & \leq e^{\left\|p^{-}\right\|}\left\|q^{-}\right\|\left\|q^{-}\right\|_{L^{1}}+e^{2\|p\|}\left\|q^{-}\right\|\left\|q^{+}\right\|_{L^{1}} \\
& +e^{2\|p\|}\left(2\|p\|_{L^{1}}\left\|q^{-}\right\|\left\|q^{+}\right\|+\|q\|_{L^{1}}\left\|q^{+}\right\|\right) \\
& \leq 2 e^{2\|p\|}\|q\|\left(\|q\|_{L^{1}}+\|p\|_{L^{1}}\|q\|\right) .
\end{aligned}
$$

In view of $(2.31)$ this completes the proof.

Remark. We notice that the estimate (2.29) is invariant under scaling. Indeed, when $\delta_{\lambda} p(x)=\lambda^{2} p(\lambda x), \lambda>0$, it follows from $(2.24)$ that $f_{\delta_{\lambda} p}(x)=\lambda^{2} f_{p}(\lambda x)$ and then

$$
\int_{-\infty}^{\infty} e^{a|y|}\left|f_{p}(y)\right| d y=\frac{1}{\lambda} \int_{-\infty}^{\infty} e^{a \lambda|y|}\left|f_{\delta_{\lambda} p}(y)\right| d y
$$

The assertion follows since when $p$ is replaced by $\delta_{\lambda} p$ and $a$ by $\lambda a$, then the right hand side of (2.29) is multiplied by $\lambda$.

Remark. It follows from the proof of Theorem 2.4, or, alternatively, directly from (2.13) that when $x \leq 0$ in the support of $p$, the estimate (2.29) improves to the following scaling invariant bound

$$
\int_{-\infty}^{0} e^{a|y|}\left|f_{p}(y)\right| d y \leq e^{\|p\|}\|p\|_{L^{1}}\|q\|
$$


The estimate (2.29) will be particularly useful when estimating the Fourier transform of $f_{p}$ at low frequencies. On the other hand, for high frequencies the following bound on $f_{p}$ is available. This bound will be needed later.

Theorem 2.5 Assume that $(1+|x|) e^{2 a|x|} p(x) \in L^{1}$ for some $a>0$. Then

$$
\int_{-\infty}^{\infty} e^{a|y|}\left|f_{p}(y)\right| d y \leq \frac{1}{a} \exp \left(\|p\|_{L^{1}} / a\right) \iint e^{2 a|x-y|}|p(x) p(y)| d x d y .
$$

Proof: Computing the Fourier transform in (2.17), we find that

$$
\hat{X}(k)=i k-\frac{1}{2} \int_{-\infty}^{\infty} p(x) e^{i x k} g(x, k) d x
$$

where $g(x, k)$ is the left Jost function, introduced in (2.8). Put $m(x, k)=e^{i x k} g(x, k)$. Then $m(x, k)$ satisfies

$$
m(x, k)=1+\int_{-\infty}^{x} D_{k}(x-t) p(t) m(t, k) d t
$$

with

$$
D_{k}(y)=\frac{1}{2 i k}\left(e^{2 i k y}-1\right)
$$

see [3]. The integral representation (2.35) can also be found in [3]. The equation (2.36) is solved by iteration,

$$
m(x, k)=1+\sum_{n=1}^{\infty} g_{n}(x, k)
$$

where

$$
g_{n}(x, k)=\int_{x_{n} \leq x_{n-1} \leq \ldots \leq x} D_{k}\left(x-x_{1}\right) \ldots D_{k}\left(x_{n-1}-x_{n}\right) p\left(x_{1}\right) \ldots p\left(x_{n}\right) d x_{1} \ldots d x_{n} .
$$

When $\operatorname{Im} k \geq-a$, we estimate $D_{k}(y)$ by $e^{2 a y} /|k|, y \geq 0$, and this gives

$$
\begin{aligned}
\left|g_{n}(x, k)\right| & \leq \frac{e^{2 a x}}{|k|^{n}} \int_{x_{n} \leq x_{n-1} \leq \ldots \leq x}\left|p\left(x_{1}\right)\right| \ldots\left|p\left(x_{n-1}\right)\right| e^{-2 a x_{n}}\left|p\left(x_{n}\right)\right| d x_{1} \ldots d x_{n} \\
& \leq \frac{e^{2 a x}}{|k|^{n}} \frac{(u(x))^{n-1}}{(n-1) !}\left(\int_{-\infty}^{x} e^{-2 a t}|p(t)| d t\right),
\end{aligned}
$$

where

$$
u(x)=\int_{-\infty}^{x}|p(t)| d t
$$

We get

$$
|m(x, k)-1| \leq \frac{e^{2 a x}}{|k|}\left(\int_{-\infty}^{x} e^{-2 a t}|p(t)| d t\right) \exp \left(\|p\|_{L^{1}} /|k|\right) .
$$


Now

$$
\hat{X}(k)=i k-\frac{1}{2}\left(\int_{-\infty}^{\infty} p(x) d x\right)-\frac{1}{2} \hat{f}_{p}(k),
$$

and using (2.35) and (2.37) we get the following bound on $\hat{f}_{p}$,

$$
\left|\hat{f}_{p}(k)\right| \leq \frac{1}{|k|} \exp \left(\|p\|_{L^{1}} /|k|\right)\left(\iint e^{2 a|x-y|}|p(x)||p(y)| d x d y\right), \quad \operatorname{Im} k \geq-a .
$$

In particular when $p \geq 0$ and $k=-i a$ is purely imaginary, then $f_{p} \geq 0$ and it follows that

$$
\int_{-\infty}^{0} e^{a|y|} f_{p}(y) d y=\left|\hat{f}_{p}(-i a)\right| \leq \frac{1}{a} \exp \left(|| p \|_{L^{1}} / a\right)\left(\iint e^{2 a|x-y|}|p(x)||p(y)| d x d y\right) .
$$

Since $\left|f_{p}\right| \leq f_{|p|}$, the proposition follows.

Remark. Using (2.24) and (2.25) we see that the estimate (2.34) is both scaling and translation invariant.

\subsection{Density of the poles}

We shall now introduce the relevant class of potentials. We say that a potential $p$ is super-exponentially decaying if $e^{2 a|x|} p(x) \in L^{1}(\mathbf{R})$ for any $a>0$. It follows then from Theorem 2.4 that when $p$ is super-exponentially decaying, $\hat{X}_{p}$ extends to an entire analytic function, and it is easily seen that $\hat{Y}_{p}$ enjoys the same property. The relation (2.4) extends to $\mathbf{C}$ as

$$
k^{2}+\hat{Y}(k) \hat{Y}(-k)=\hat{X}(k) \hat{X}(-k)
$$

since $\overline{\hat{X}(k)}=\hat{X}(-k), \overline{\hat{Y}(k)}=\hat{Y}(-k), k \in \mathbf{R}$. The zeros of $\hat{X}(k)$ in $\operatorname{Im} k<0$ will be called scattering poles or resonances. These are the poles of the transmission coefficient $t(k)=i k / \hat{X}(k)$ in $\mathbf{C}_{-}$. It follows from (2.40) that the scattering poles coincide with the poles of the reflection coefficient

$$
r(k)=\frac{\hat{Y}(k)}{\hat{X}(k)}
$$

in $\mathbf{C}_{-}$. When $p$ decays at some fixed exponential rate, the continuation of $\hat{X}$ can be made to a strip $S$ around the real axis. In this case, the scattering poles are the zeros of $\hat{X}$ in $S_{-}=\{k \in S ; \operatorname{Im} k<0\}$.

Theorems 2.4 and 2.5 have a direct application to the problem of estimating the density of resonances. We introduce the counting function $N(r)$ as the number of scattering poles in the disc $|k| \leq r$, counted with their multiplicities. In the case of compactly supported potentials it was proved by Zworski [17] that

$$
N(r)=\frac{2 d}{\pi} r+o(r), \quad r \rightarrow \infty
$$


where $d$ is the diameter of the support of the potential. For some special class of superexponentially decaying potentials and using different methods, Froese [5] established that

$$
N(r)=C r^{\rho}+o\left(r^{\rho}\right),
$$

where $\rho$ is the order of growth of the Fourier transform of the potential. Here we shall give an upper bound on $N(r)$ for a general super-exponentially decaying potential. Similar bounds in any odd dimension have been obtained by Froese [6]. We shall nevertheless give a proof, as it is short and serves as a preparation for the more general results to follow.

The bound on $N(r)$ will be given in terms of the function

$$
\varphi_{p}(r)=\log \left(\iint e^{2 r|x-y|}|p(x) p(y)| d x d y\right), \quad r \geq 0 .
$$

We notice that this is a strictly increasing convex function, with linear growth at infinity if and only if $p$ is compactly supported.

Theorem 2.6 Let $p$ be a super-exponentially decaying potential. Then

$$
\int_{r / 2}^{r} \frac{N(t)}{t} d t \leq C+\varphi_{p}(r), \quad r \geq 1
$$

for some $C$ depending on $p$.

Proof: First, we shall estimate the growth of $\hat{X}_{p}$ in the lower half-plane. By (2.39) we have

$$
\left|\hat{f}_{p}(k)\right| \leq \frac{1}{|k|} \exp \left(|| p \|_{L^{1}} /|k|\right) \iint e^{2|\beta(x-y)|}|p(x) p(y)| d x d y,
$$

when $k=\alpha+i \beta$. Then

$$
\left|\hat{f}_{p}(k)\right| \leq C(p) e^{\varphi_{p}(|\beta|)}, \quad|k|=r \geq 1
$$

Here $C(p)$ denotes different constants, depending on the potential. Since $\hat{X}_{p}(k)=$ $i k-(1 / 2)\left(\int p\right)-(1 / 2) \hat{f}_{p}(k)$, we have that a similar bound holds for $\hat{X}_{p}$,

$$
\left|\hat{X}_{p}(k)\right| \leq C(p) \exp \left(\varphi_{p}(r)\right), \quad|k| \leq r .
$$

Assume now that $\hat{X}_{p}(0) \neq 0$. Then Jensen's formula, see [16],

$$
\int_{0}^{r} \frac{N(t)}{t} d t=\frac{1}{2 \pi} \int_{-\pi}^{\pi} \log \left|\hat{X}_{p}\left(r e^{i \theta}\right)\right| d \theta-\log \left|\hat{X}_{p}(0)\right|
$$


together with (2.44) implies (2.43) at once. When $\hat{X}_{p}(0)=0$, we use the fact that the zero is of order one, since $\left|\hat{X}_{p}(k)\right|^{2} \geq k^{2}, k \in \mathbf{R}$. Then we can apply the preceding argument to $\hat{X}_{p}(k) / k$, the conclusion being the same.

Remark. Since when $a>1$ we have

$$
N(r)=(\log a)^{-1} N(r) \int_{r}^{a r} \frac{d t}{t} \leq(\log a)^{-1} \int_{r}^{a r} \frac{N(t)}{t} d t
$$

we also get a bound on $N(r)$.

\section{Scattering poles near the real axis}

\subsection{Pole-free regions for compactly supported potentials}

As a preparation for the later considerations, and also, since some of the results are only valid in this case, we shall work here with compactly supported integrable potentials.

Let $[a, b]$ be the smallest interval, containing the support of the potential $p$. Then it follows from (2.20) and (2.21) that

$$
\operatorname{supp} X \subseteq[-2(b-a), 0], \quad \operatorname{supp} Y \subseteq[2 a, 2 b],
$$

and therefore $\hat{X}$ and $\hat{Y}$ are entire functions. Moreover, it was proved by Zworski [17] that $[-2(b-a), 0]$ is the smallest interval containing the support of $X$. The functions $f(x, k)$ and $g(x, k)$ are also entire analytic functions of $k$. We have

$$
f(x, k)=e^{i x k} \quad \text { for } \quad x>b,
$$

and

$$
g(x, k)=e^{-i x k} \quad \text { for } \quad x<a .
$$

Recall that the scattering poles, or resonances of $p$, are defined as the points $k \in \mathbf{C}_{-}$, for which $\hat{X}(k)=0$. From (2.19) it follows that the poles can be characterized in the following way: $k \in \mathbf{C}_{-}$is a scattering pole if and only if there exists a function $\varphi(x)$ such that

$$
-\varphi^{\prime \prime}(x)+p(x) \varphi(x)=k^{2} \varphi(x)
$$

and

$$
\varphi(x)=\left\{\begin{array}{cc}
A e^{i x k} & \text { when } \quad x>b \\
e^{-i x k} & x<a .
\end{array}\right.
$$

for some number $A$. The solution $\varphi(x)=g(x, k)$ grows exponentially at $\pm \infty$. Let us also notice that the poles are symmetric with respect to reflection in the imaginary axis. Indeed, we have that

$$
\overline{\hat{X}(k)}=\hat{X}(-\bar{k}), \quad k \in \mathbf{C},
$$


since

$$
\overline{\hat{X}(k)}=\hat{X}(-k), \quad k \in \mathbf{R} .
$$

Some lower bounds on the imaginary part of the poles ouside the imaginary axis, depending on the real part, were obtained in the works [7] and [4]. Here we shall be concerned with the existence of a pole-free strip below the real axis.

Theorem 3.1 Let $p \in L^{1}(\mathbf{R})$ be supported by an interval of length $d>0$. Define the function

$$
h(p)=\left(\frac{1}{4 d}\right) e^{-2 d\|p\|_{L^{1}}}
$$

Then we have

1. The set $S=\{k ;-h(p)<\operatorname{Im} k<0$, Re $k \neq 0\}$ contains no scattering poles of $p$.

2. If ed\| $\|p\|_{L^{1}}<1$ then the interval $-i\left(0,-(1 / 2 d) \log \left(d\|p\|_{L^{1}}\right)\right)$ contains at most one pole of $p$.

3. Assume that $p \geq 0$. Then the strip

$$
S=\{k ;-h(p)<\operatorname{Im} k<0\}
$$

contains at most one pole of $p$. Moreover, set

$$
g(p)=\min \left(h(p), \frac{\|p\|_{L^{1}}}{2}\right) .
$$

Then the strip

$$
\Sigma=\{k \in \mathbf{C},-g(p)<\operatorname{Im} k<0\}
$$

is a pole-free region.

Remark. It follows from Theorem 3.1 that, as a sequence of nonnegative potentials tends to infinity, the scattering poles can approach the real axis at most exponentially fast.

The following proposition gives the first part of the theorem.

Proposition 3.2 Let $p \in L^{1}(\mathbf{R})$ be supported by an interval of length $d>0$. Assume that $k$ is a scattering pole of $p$ with $\operatorname{Re} k \neq 0$. Then,

$$
d|\operatorname{Im} k| \geq \frac{1}{4} e^{-2 d\|p\|_{L^{1}}}
$$


Proof: By (2.24) and (2.25) we know that the scattering poles are invariant under translations of the potential, and $k$ is a pole of $p$ if and only if $\lambda k$ is a pole of $\delta_{\lambda} p(x)=$ $\lambda^{2} p(\lambda x)$. Therefore we may assume that the support of $p$ is contained in the interval $[0,1]$. The function $\varphi(x, k)$ satisfies

$$
-\varphi^{\prime \prime}(x, k)+p(x) \varphi(x, k)=k^{2} \varphi(x, k),
$$

and

$$
\varphi^{\prime}(0)=-i k \varphi(0), \quad \varphi^{\prime}(1)=i k \varphi(1) .
$$

Multiplying (3.4) by $\overline{\varphi(x, k)}$ and integrating by parts, we get

$$
\begin{aligned}
k^{2} & \int_{0}^{1}|\varphi(x, k)|^{2} d x=\int_{0}^{1}\left(-\varphi^{\prime \prime}(x, k) \overline{\varphi(x, k)}+p(x)|\varphi(x, k)|^{2}\right) d x \\
= & -i k\left(|\varphi(0)|^{2}+|\varphi(1)|^{2}\right)+\int_{0}^{1}\left(\left|\varphi^{\prime}(x, k)\right|^{2}+p(x)|\varphi(x, k)|^{2}\right) d x .
\end{aligned}
$$

Therefore,

$$
-\operatorname{Re} k\left(|\varphi(0)|^{2}+|\varphi(1)|^{2}\right)=2 \operatorname{Re} k \operatorname{Im} k \int_{0}^{1}|\varphi(x, k)|^{2} d x,
$$

and, as $\operatorname{Re} k \neq 0$, we get

$$
|\operatorname{Im} k|=\frac{|\varphi(0)|^{2}+|\varphi(1)|^{2}}{2 \int_{0}^{1}|\varphi(x, k)|^{2} d x .}
$$

We shall now estimate $\varphi(x, k)=g(x, k)$ when $x \in[0,1]$. We may write

$$
g(x, k)=e^{-i x k}+\int_{-\infty}^{x} R(x, y) e^{-i y k} d y .
$$

It follows from $(2.15)$ that $R(x, y) \neq 0$ if and only if $-x \leq y \leq x$. Therefore, writing $\beta=\operatorname{Im} k$, we get

$$
\begin{aligned}
& |g(x, k)| \leq e^{-x|\beta|}+\int_{-x}^{x}|R(x, y)| e^{-y|\beta|} d y \\
& \quad \leq 1+e^{x|\beta|} \int_{-x}^{x}|R(x, y)| d y \leq e^{|\beta|}\left(1+\int_{-x}^{x}|R(x, y)| d y\right) .
\end{aligned}
$$

Using the estimate (2.13), we get

$$
\int_{-x}^{x}|R(x, y)| d y \leq e^{v(x)} \int_{0}^{x} u(t) e^{-v(t)} d t=e^{v(x)-v(0)}-1=e^{v(x)}-1,
$$

since $v(0)=0$. Therefore,

$$
|g(x, k)| \leq e^{|\beta|+v(x)}, \quad 0<x<1 .
$$


Since $v$ is increasing and $v(1)=\int_{0}^{1}(1-t)|p(t)| d t \leq\|p\|_{L^{1}}$, we get

$$
\int_{0}^{1}|g(x, k)|^{2} d x \leq e^{2|\beta|+2|| p \|_{L^{1}}} .
$$

As $|g(0, k)|^{2}=1$, it follows from (3.6) and (3.8) that

$$
|\beta| \geq \frac{1}{2 e^{2|\beta|+2|| p \|_{L^{1}}} .}
$$

Hence if $s=2|\beta|$ and $t=2\|p\|_{L^{1}}$, we have $s e^{s} \geq e^{-t}$. If $\sigma=e^{-t} / 2$ we have

$$
\sigma e^{\sigma}=\frac{1}{2} e^{-t} \exp \left(e^{-t} / 2\right) \leq \frac{e^{1 / 2}}{2} e^{-t} \leq e^{-t} \leq s e^{s} .
$$

Hence $\sigma \leq s$, i.e.

$$
|\operatorname{Im} k| \geq \frac{1}{4} e^{-2\|p\|_{L^{1}}}
$$

This completes the proof.

The second part of the theorem is given in the following proposition.

Proposition 3.3 Let $p \in L^{1}$ be supported by an interval of length d and assume that ed $\|p\|_{L^{1}}<1$. Then the interval $-i\left(0,-(1 / 2 d) \log \left(d\|p\|_{L^{1}}\right)\right)$ contains at most one pole of $p$.

Proof: When proving the proposition we may assume that $\operatorname{supp}(p) \subset[-1,0]$ in view of (2.24) and (2.25). Consider

$$
\varphi(\lambda)=\hat{X}_{p}(-i \lambda)=\lambda-\frac{1}{2}\left(\int p(y) d y\right)-\frac{1}{2} \int_{-\infty}^{0} f_{p}(y) e^{\lambda|y|} d y, \quad \lambda \geq 0,
$$

where

$$
f_{p}(y)=\int_{-\infty}^{\infty} p(z) R_{p}(z, z+y) d z .
$$

Since $y \in[-2,0]$ in $\operatorname{supp}\left(f_{p}\right)$, using $(2.33)$ we obtain

$$
\begin{aligned}
& \int_{-\infty}^{0}|y|\left|f_{p}(y)\right| e^{\lambda|y|} d y \leq 2 \int_{-\infty}^{0}\left|f_{p}(y)\right| e^{\lambda|y|} d y \\
\leq & 2 e^{\|p\|}\|p\|_{L^{1}} \int_{-\infty}^{\infty} e^{2 \lambda|x|}|x||p(x)| d x \leq 2 e^{\|p\|_{L^{1}}+2 \lambda}\|p\|_{L^{1}}^{2} .
\end{aligned}
$$

Therefore if $e\|p\|_{L^{1}}<1$ we have

$$
\int_{-\infty}^{0}|y|\left|f_{p}(y)\right| e^{\lambda|y|} d y \leq 2 e^{2 \lambda}\|p\|_{L^{1}},
$$


and then

$$
\begin{aligned}
\varphi^{\prime}(\lambda) & =1-\frac{1}{2} \int_{-\infty}^{0}|y| e^{\lambda|y|} f_{p}(y) d y \geq 1-\frac{1}{2} \int_{-\infty}^{0}|y| e^{\lambda|y|}\left|f_{p}(y)\right| d y \\
& \geq 1-e^{2 \lambda}\|p\|_{L^{1}}>0
\end{aligned}
$$

if $\lambda \in\left(0,-(1 / 2) \log \left(\|p\|_{L^{1}}\right)\right)$. Therefore $\varphi(\lambda)$ has at most one zero in this interval, and the proof is complete.

It remains to prove the third assertion in the theorem. In the case of nonnegative potentials additional information on the purely imaginary poles is available.

Proposition 3.4 Let $p \in L^{1}$ be super-exponentially decaying and nonnegative. Then $H_{p}$ can have at most two poles on the imaginary axis. If $k$ is such a pole, then

$$
|k|>\eta_{0}(p) \equiv(1 / 2)\|p\|_{L^{1}}+(1 / 2) \int_{-\infty}^{\infty} p(x)\|R(x, \cdot)\|_{L^{1}} d x
$$

Define the functions

$$
\eta_{1}(p)=\sup \left\{\lambda>0 ; \int_{-\infty}^{\infty}|y| f_{p}(y) e^{\lambda|y|} d y<2\right\} \in[-\infty, \infty)
$$

and

$$
\eta(p)=\max \left(\eta_{0}(p), \eta_{1}(p)\right),
$$

where $f_{p}(y)$ is defined in (2.28). Then there can be at most one pole of the form $-i \lambda$, where $\lambda \in(0, \eta(p))$.

Finally, if $p$ is such that

$$
\int_{-\infty}^{\infty}|y| f_{p}(y) d y \geq 2
$$

then $H_{p}$ has no purely imaginary poles.

Proof: We may assume that $p$ is not identically zero. Since

$$
f_{p}(y)=\int_{-\infty}^{\infty} p(z) R_{p}(z, z+y) d z \geq 0
$$

it follows that the function

$$
\varphi(\lambda)=\hat{X}_{p}(-i \lambda)=\lambda-\frac{1}{2}\left(\int p(y) d y\right)-\frac{1}{2} \int_{-\infty}^{0} f_{p}(y) e^{\lambda|y|} d y, \quad \lambda \geq 0,
$$

is concave for $\lambda \geq 0$, and $\varphi(0)<0$. Therefore, there can be at most two zeros on $(0, \infty)$, and if $\varphi(\lambda)=0$, then

$$
2 \lambda>\|p\|_{L^{1}}+\int_{-\infty}^{\infty} f_{p}(y) d y=\|p\|_{L^{1}}+\int_{-\infty}^{\infty} p(x)\|R(x, \cdot)\|_{L^{1}} d x,
$$


which is (3.9). Also, if $\varphi^{\prime}(0) \leq 0$, then $\varphi(\lambda)<0$ for $\lambda>0$, and this gives (3.10).

Finally, we shall estimate the length of an interval, containing at most one purely imaginary pole of $H_{p}$. Since $\varphi^{\prime}(\lambda)>0$ on $\left(0, \eta_{1}(p)\right)$, it follows that $\varphi(\lambda)$ has at most one zero in this interval. Combining this with (3.9) completes the proof.

Remark. Let $H_{\alpha p}=-D^{2}+\alpha p$, where $p \geq 0$ is some fixed super-exponentially decaying potential, and $\alpha>0$ is the coupling constant. Using the arguments of Proposition 3.4, we may draw the following conclusions, concerning the behaviour of the purely imaginary poles as functions of $\alpha$. When $\alpha=0$, then $\hat{X}(0)=0$. When $\alpha>0$ is sufficiently small, the function $\varphi(\lambda)=\varphi_{\alpha}(\lambda)$ has exactly two zeros $\lambda_{1}(\alpha)$ and $\lambda_{2}(\alpha)$, with $\lambda_{1}(\alpha)$ close to 0 , and $\lambda_{2}(\alpha)$ close to $+\infty$. As $\alpha$ grows, the distance between the two poles decreases. For a certain value of the coupling constant $\alpha_{0}$, the poles meet, the function $\varphi_{\alpha_{0}}$ having a zero of multiplicity two. Increasing the coupling constant further results in splitting of the double root, and the poles leave the imaginary axis, so that (3.2) is respected.

We shall now complete the proof of Theorem 3.1. It follows from Propositions 3.2 and 3.4 that when $p \geq 0$, then the strip

$$
\Sigma=\{k \in \mathbf{C},-g(p)<\operatorname{Im} k<0\}
$$

is a pole-free region. We have to consider therefore

$$
S=\{k \in \mathbf{C},-h(p)<\operatorname{Im} k<0\} .
$$

From Proposition 3.2 we know that $S$ contains no poles off the imaginary axis, and we only have to prove that the interval $(0, h(p))$ contains at most one $\lambda$ such that $-i \lambda$ is a resonance.

Let $\eta_{0}(p)$ and $\eta_{1}(p)$ be defined as in Proposition 3.4, and consider

$$
\eta(p)=\max \left(\eta_{0}(p), \eta_{1}(p)\right) .
$$

We know that the interval $-i(0, \eta(p))$ contains at most one pole, and it suffices therefore to prove that $\eta(p) \geq h(p)$. When doing this, we may again assume that $\operatorname{supp}(p) \subset$ $[-1,0]$, see $(2.24)$ and $(2.25)$. It follows then as in Proposition 3.3 that

$$
\int_{-\infty}^{0}|y| f_{p}(y) e^{\lambda|y|} d y \leq 2 e^{2 \lambda+\|p\|_{L^{1}}}\|p\|_{L^{1}}^{2} .
$$

We write $t=\|p\|_{L^{1}}$, so that $\eta_{0}(p) \geq(1 / 2) t$ and $\eta_{1}(p) \geq \lambda$ if $e^{2 \lambda+t} t^{2}=1$. Then the inequality $\eta(p) \geq h(p)$ holds if

$$
\frac{1}{2 e^{2 t}} \leq \max \left(t, \log \left(\frac{e^{-t}}{t^{2}}\right)\right) .
$$

It is enough to prove this when $1 /(2 \exp (2 t))>t$. But then we have

$$
\log \left(\frac{e^{-t}}{t^{2}}\right)>\log \left(4 e^{-t} e^{4 t}\right)>1>\frac{1}{2 e^{2 t}} .
$$

The proof of Theorem 3.1 is now complete. 


\subsection{A pole-free strip for exponentially decaying potentials}

In the beginning of this section it will be assumed that the potential $p$ is such that

$$
(1+|x|) e^{2 a|x|} p(x) \in L^{1}
$$

for some $a>0$. It follows from Theorem 2.4 that $\hat{X}_{p}$ is analytic in $\operatorname{Im} k>-a$ and continuous up to the boundary of this set. We shall study the location of the scattering poles near the real axis. In particular, we shall be interested in estimates that are uniform in $p$.

When $q \geq 0$ is such that $(1+|x|) e^{2 a|x|} q(x) \in L^{1}$, set

$$
B_{q}=\left\{p \in L^{1}(\mathbf{R}) ;|p| \leq q\right\} .
$$

If $\mu$ is a measure, such that $|\mu| \leq q$, where $q \in L_{\text {loc }}^{1}$, then $\mu$ is absolutely continuous. The following result is then immediate from the first part of the proof of Theorem 2.2.

Proposition 3.5 Let $p_{j}$ be a sequence in $B_{q}$. Then there is a subsequence $p_{j_{k}}$ and some $p \in B_{q}$ such that $p_{j_{k}} \rightarrow p$ weakly in $L^{1}(\mathbf{R})$.

We have the following

Theorem 3.6 Let $K$ be a compact set in $\operatorname{Im} k>-a$ and $F \subset L^{1}(\mathbf{R})$ be sequentially closed in the weak topology of measures. Assume that there is an integer $n$ such that $\hat{X}_{p}$ has at most $n$ zeros in $K$ when $p \in B_{q} \cap F$. Then there is an open neighbourhood $\Omega$ of $K$ such that $\hat{X}_{p}$ has at most $n$ zeros in $\Omega$ when $p \in B_{q} \cap F$. (All zeros are counted with multiplicities.)

Proof: We write $K=\cap_{j=1}^{\infty} \Omega_{j}$, where the $\Omega_{j}$ form a decreasing sequence of small open neighbourhoods of $K$. Assume that the statement is false. Then we may for every $j$ find $p_{j} \in B_{q} \cap F$ such that $\hat{X}_{j}=\hat{X}_{p_{j}}$ has at least $n+1$ zeros in $\Omega_{j}$. Passing to a subsequence, we may assume that $p_{j} \rightarrow p$ weakly in $L^{1}$, where $p \in B_{q} \cap F$. Then we know by Theorem 2.2 that $\hat{X}_{j}$ converges uniformly to $\hat{X}_{p}$ in the upper half-plane, and since the sequence $\hat{X}_{j}$ is bounded in the space of functions, analytic in the set $\operatorname{Im} k>-a$, it follows by a normal families argument that $\hat{X}_{j} \rightarrow \hat{X}_{p}$ locally uniformly in $\operatorname{Im} k>-a$. Take now a relatively compact open neighbourhood $V$ of $K$ such that $\hat{X}_{p} \neq 0$ on $\partial V$, and all zeros of $\hat{X}_{p}$ in $V$ are contained in $K$. An application of the argument principle to $V$ gives that $\hat{X}_{p}$ must have as many zeros as $\hat{X}_{j}$ in $V$ when $j$ is large and we get a contradiction.

As an application of Theorem 3.6 we get

Proposition 3.7 There exists an open complex neighbourhood of the origin $V$, such that $V$ contains at most one pole of any $p \in B_{q}$. 
Proof: Since $B_{q}$ is sequentially closed in the weak topology of measures, the result follows from the fact that when $p \in B_{q}$, then $\hat{X}_{p}$ vanishes at most to the first order at the origin, since

$$
\left|\hat{X}_{p}(k)\right|^{2} \geq k^{2}, \quad k \in \mathbf{R},
$$

in view of (2.4) and (2.16).

Using (3.12) together with Proposition 3.7 it is not difficult to see that there exists a strip of the form $-\lambda(q)<\operatorname{Im} k<0$ which contains at most one pole of any $p \in B_{q}$. Due to the symmetry of the poles, such a pole is then situated on the imaginary axis. Now if $p \in B_{q}$ is nonnegative and $k$ is a purely imaginary pole of $p$, we have that $|k| \geq\|p\|_{L^{1}} / 2$ in view of Proposition 3.4. It follows therefore that if $0 \leq p \in B_{q}$ is such that $\|p\|_{L^{1}}$ is sufficiently small, then the strip $\left\{k ;-\|p\|_{L^{1}} / 2<\operatorname{Im} k<0\right\}$ contains no poles of $p$. We shall now prove a more precise result.

Theorem 3.8 Assume that $0 \leq p$ is super-exponentially decaying and that

$$
\inf _{x} \int_{-\infty}^{\infty} e^{\|p\|_{L^{1}}|x-y| / 2}|x-y| p(y) d y \leq \frac{1}{10} .
$$

Then $H_{p}$ has no resonances in the strip $S=\left\{k ;-\|p\|_{L^{1}} / 4 \leq \operatorname{Im} k<0\right\}$.

Proof: Since the position of the resonances is not changed when $p$ is replaced by a translate of $p$ we may assume that

$$
\int_{-\infty}^{\infty} e^{\|p\|_{L^{1}}|y| / 2}|y| p(y) d y \leq \frac{1}{10}
$$

Also, since the conditions and conclusions of the theorem are the same for $p(x)$ and $\delta_{\lambda} p(x)=\lambda^{2} p(\lambda x)$, we may assume that $\|p\|_{L^{1}}=1$. We notice that

$$
\begin{aligned}
\operatorname{Re} \hat{X}_{p}(\beta-i \lambda) & =\lambda-\frac{1}{2}-\frac{1}{2} \int_{-\infty}^{0} f_{p}(y) e^{\lambda|y|} \cos (\beta|y|) d y \\
& \leq \lambda-\frac{1}{2}+\frac{1}{2} \int_{-\infty}^{0} f_{p}(y) e^{\lambda|y|} d y \leq-\frac{1}{4}+\frac{1}{2} \int_{-\infty}^{0} f_{p}(y) e^{\lambda|y|} d y
\end{aligned}
$$

when $\beta \in \mathbf{R}$ and $\lambda \leq 1 / 4$. It suffices to prove therefore that

$$
\int_{-\infty}^{0} f_{p}(y) e^{|y| / 4} d y<\frac{1}{2}
$$

Set $q(y)=e^{|y| / 2} p(y)$. An application of Theorem 2.4 gives

$$
\int_{-\infty}^{0} f_{p}(y) e^{|y| / 4} d y \leq 2 e^{2\|p\|}\|q\|\left(\|q\|_{L^{1}}+\|q\|\|p\|_{L^{1}}\right) .
$$


By our assumptions we have $\|p\|_{L^{1}}=1$, and $\|p\| \leq\|q\| \leq 1 / 10$. Hence

$$
\int_{-\infty}^{0} e^{|y| / 4} f_{p}(y) d y \leq 2 e^{2 / 10} \frac{1}{10}\left(\|q\|_{L^{1}}+\frac{1}{10}\right) .
$$

We have

$$
\|q\|_{L^{1}} \leq\|q\|+\int_{|y| \leq 1} e^{|y| / 2} p(y) d y \leq \frac{1}{10}+e^{1 / 2}\|p\|_{L^{1}}=\frac{1}{10}+e^{1 / 2} .
$$

We have thus proved that

$$
\begin{aligned}
\int_{-\infty}^{0} e^{|y| / 4} f_{p}(y) d y & \leq \frac{2}{10} e^{2 / 10}\left(\frac{2}{10}+e^{1 / 2}\right) \\
& \leq \frac{2}{10}\left(1+\frac{2}{10}+\left(\frac{2}{10}\right)^{2}\right)\left(\frac{2}{10}+\frac{18}{10}\right) \\
& =\frac{4}{10}(1,24)=0.496<\frac{1}{2}
\end{aligned}
$$

This completes the proof.

Remarks:

1. It follows from Theorem 3.1 that a similar result holds for compactly supported nonnegative potentials, the condition similar to (3.13) in this case being $d\|p\|_{L^{1}} \leq t_{0} / 2$, where $d$ is the length of the support of the potential and $t_{0} e^{t_{0}}=1$.

2. Using the arguments, similar to those used in the proof of Theorem 3.8 together with the estimate (3.12) it is straightforward to estimate the width of a pole-free strip in the case when $p$ is not small. This leads, however, to more complicated expressions, and therefore we shall avoid stating them explicitly.

\subsection{Density of resonances in strips}

We shall now turn to the problem of estimating the number of scattering poles in arbitrary strips. We notice that any super-exponentially decaying potential $p$ has only finitely many poles in $\operatorname{Im} k \geq-a$ for any $a>0$. In fact, the function $\hat{X}_{p}$ is entire analytic and $\left|\hat{X}_{p}(k) / k\right| \rightarrow 1$ when $|k| \rightarrow \infty, \operatorname{Im} k+a \geq 0$. We let $N_{p}(a)$ denote the number of scattering poles in the set $\operatorname{Im} k \geq-a$. Our goal is to find upper bounds on $N_{p}(a)$.

Our starting point is the following general result. The proof that we shall give has been communicated to the author by Professor Lars Hörmander. The original proof of the author was different and gave a slightly weaker result. The author is grateful to Professor Hörmander for this contribution and for referring to [11], where more general results have been given. 
Proposition 3.9 Let $h$ be a function, analytic in a neighbourhood of the set $\operatorname{Im} k \geq 0$. Assume that $|h(k)| \leq 1$ along $\mathbf{R}$ and

$$
h(k)=1+\frac{\gamma}{i k}+O\left(\frac{1}{|k|^{1+\delta}}\right), \quad|k| \rightarrow \infty, \quad \operatorname{Im} k \geq 0,
$$

for some $\delta>0$. Then $\gamma \geq 0$ and if $k_{j}$ are the zeros of $h$ in the upper half-plane, repeated according to their multiplicity, we have

$$
\gamma=2 \sum \operatorname{Im} k_{j}-\frac{1}{\pi} \int_{-\infty}^{\infty} \log |h(t)| d t \geq 2 \sum \operatorname{Im} k_{j}
$$

Proof: It follows from the assumptions and the maximum principle that $|h(k)| \leq 1$ when $\operatorname{Im} k \geq 0$, and since

$$
|h(k)|=1+\operatorname{Re}\left(\frac{\gamma}{i k}\right)+o\left(|k|^{-1}\right),
$$

we must have $\operatorname{Re}(\gamma / i k) \leq 0$ when $\operatorname{Im} k>0$, thus $\gamma \geq 0$.

The Riesz representation formula for functions, subharmonic and $\leq 0$ in the upper half-plane (see [10]) gives

$$
\log |h(k)|=a \operatorname{Im} k+\frac{\operatorname{Im} k}{\pi} \int_{-\infty}^{\infty} \frac{\log |h(t)|}{|t-k|^{2}} d t+\sum \log \left|\frac{k-k_{j}}{k-\overline{k_{j}}}\right|, \quad \operatorname{Im} k>0,
$$

where $k_{j}$ are the finitely many zeros of $h$ in $\operatorname{Im} k>0$. The left-hand side is $\operatorname{Re}(\gamma / i k)+$ $o\left(|k|^{-1}\right)$ at infinity and we have

$$
\log \left|\frac{k-k_{j}}{k-\overline{k_{j}}}\right|=\log \left|1-\frac{2 i \operatorname{Im} k_{j}}{k-\overline{k_{j}}}\right|=\operatorname{Re}\left(\frac{2 \operatorname{Im} k_{j}}{i k}\right)+O\left(|k|^{-2}\right) .
$$

Since $\log |h(t)|$ is locally integrable and $\log |h(t)|=O\left(|t|^{-1-\delta}\right)$ at infinity, we have that $\log |h(t)| \in L^{1}(\mathbf{R})$. When $\alpha \leq \arg k \leq \pi-\alpha, \alpha>0$, we have that $|k|^{2} \leq C_{\alpha}|t-k|^{2}$ for $t \in \mathbf{R}$, and therefore by Lebesgue's theorem we obtain

$$
|k|^{2} \int_{-\infty}^{\infty} \frac{\log |h(t)|}{|t-k|^{2}} d t \rightarrow \int_{-\infty}^{\infty} \log |h(t)| d t, \quad k \rightarrow \infty, \quad \alpha \leq \arg k \leq \pi-\alpha .
$$

We have that $\operatorname{Im} k /|k|^{2}=\operatorname{Im}(1 / \bar{k})=-\operatorname{Re}(1 / i k)$ and then it follows from (3.17) that the constant $a=0$ and (3.16) is true.

Corollary 3.10 If $h$ is analytic with $|h| \leq 1$ in the open upper half-plane and (3.15) holds there, then

$$
2 \sum \operatorname{Im} k_{j} \leq \gamma
$$

Thus the number of zeros with $\operatorname{Im} k_{j} \geq a / 2$ does not exceed $\gamma / a$. 
Proof: It suffices to apply the proposition to $h(k+i \varepsilon)$ for $\varepsilon>0$ and let $\varepsilon \rightarrow 0$.

We are now ready to state

Theorem 3.11 For any super-exponentially decaying $p$ we have

$$
N_{p}(a / 2) \leq C(p)\left(1+\eta_{a}(p)\right), \quad a \geq 1,
$$

where

$$
\eta_{a}(p)=\iint e^{2 a|x-y|}|p(x) p(y)| d x d y
$$

and $C(p)$ is some constant depending on $\|p\|_{L^{1}}$, but not on a.

Proof: We shall pass to a new function $F(k)$, having the same zeros as $\hat{X}_{p}$ and with the property that $|F(k)| \leq 1$ on $\operatorname{Im} k=-a$. This will make it possible to apply Proposition 3.9 to the function $F(k-i a)$.

When constructing the function $F$ we write

$$
\hat{X}_{p}(k)=i k-\frac{1}{2}\left(\int_{-\infty}^{\infty} p(x) d x\right)-\frac{1}{2} \hat{f}_{p}(k) .
$$

By (2.39) we have

$$
\left|\hat{f}_{p}(k)\right| \leq \frac{1}{|k|} \exp \left(\|p\|_{L^{1}} /|k|\right) \eta_{a}(p), \quad \operatorname{Im} k \geq-a .
$$

Using this, we shall now estimate $\hat{X}_{p}(k)$ on the line $\operatorname{Im} k=-a$. Writing $k=\xi-i a$ and using (3.18), we obtain

$$
\left|\hat{X}_{p}(k)\right|^{2} \leq \xi^{2}+c^{2}+\frac{\eta_{a}^{2}(p)}{|k|^{2}} e^{2\|p\|_{L^{1}} /|k|}+e^{\|p\|_{L^{1}} /|k|} \eta_{a}(p)\left(1+\frac{|c|}{|k|}\right),
$$

where $c=a-(1 / 2) \int p(x) d x$. Therefore, since $a \geq 1$,

$$
\begin{aligned}
\left|\hat{X}_{p}(k)\right|^{2} & \leq \xi^{2}+a^{2}+C(p) a+C(p) \eta_{a}(p)\left(1+\eta_{a}(p)\right) \\
& \leq|k|^{2}+C(p) a\left(1+\eta_{a}(p)\right)^{2}
\end{aligned}
$$

where here and in what follows we let $C(p)$ denote different constants $\geq 1$, depending only on $\|p\|_{L^{1}}$. It follows that if we take $\mu$ equal to

$$
\mu=C(p) a\left(1+\eta_{a}(p)\right)
$$

then

$$
\left|\frac{\hat{X}_{p}(k)}{i k-\mu}\right| \leq 1, \quad \operatorname{Im} k=-a
$$


since we may assume that $\mu-2 a \geq 1+\eta_{a}(p)$.

Therefore the function

$$
F(k)=\frac{\hat{X}_{p}(k)}{i k-\mu}
$$

has the same zeros as $\hat{X}_{p}$ in the set $\operatorname{Im} k+a \geq 0$, and satisfies $|F(k)| \leq 1$ on $\operatorname{Im} k=-a$. Moreover, since by (2.39)

$$
\frac{\hat{X}_{p}(k)}{i k}=1-\frac{1}{2 i k}\left(\int_{-\infty}^{\infty} p(x) d x\right)+O\left(\frac{1}{k^{2}}\right),
$$

it follows that

$$
F(k)=1+\frac{\gamma}{i k}+O\left(\frac{1}{k^{2}}\right), \quad|k| \rightarrow \infty, \quad \operatorname{Im} k \geq-a,
$$

where

$$
\gamma=\mu-(1 / 2) \int_{-\infty}^{\infty} p(x) d x
$$

and we may assume that $\gamma>0$. An application of Proposition 3.9 to the function $F(k-i a)$ shows that $N_{p}(a / 2) \leq \gamma / a$, and this completes the proof.

Remark. It is known that the scattering poles of an integrable compactly supported potential lie below a logarithmic curve, i.e. if $k$ is a pole, then

$$
|\operatorname{Im} k| \geq a+b \log |k|,
$$

with $a \in \mathbf{R}$ and $b>0$-see Theorem 3.14 and also [12], where this result is proved in a more general setting in the three-dimensional case. Comparing Theorem 2.6 and Theorem 3.11 we see, in particular, that the latter reflects the logarithmic bound (3.22).

Remark. We notice that after obvious modifications, the results above are also valid when the potential $p$ decays at some fixed exponential rate.

We shall finish this section by making some remarks concerning the question of existence of resonances of exponentially decaying potentials. It is well known that in the one-dimensional case, any compactly supported potential has infinitely many resonances-see [17] for the precise results, and in [15] this is established for smooth potentials in any odd dimension. However, the situation is completely different for potentials, decaying at some fixed exponential rate. This is already seen from the existence of the reflectionless potentials, all the resonances in this case being square roots of the eigenvalues. We shall now give an example of an exponentially decaying potential without bound states, which has only finitely many resonances in the set where these are naturally defined. We start with the right reflection coefficient

$$
r(k)=\frac{2}{(k+i)(k+2 i)},
$$


and try to find the corresponding potential $p$, which has no bound states. Then we must have that $x \leq 0$ in the support of the inverse Fourier transform of $r$, and it follows from the Gelfand-Levitan equation for the right scattering data that $x \leq 0$ in $\operatorname{supp}(p)$, see [14]. In order to find the potential on the negative half-axis, we first compute the left reflection coefficient $\rho(k)$. We have the well-known formulas, see [13]

$$
t(k)=\exp \left(\frac{1}{2 \pi i} \int_{-\infty}^{+\infty} \frac{\log \left(1-|r(\lambda)|^{2}\right)}{\lambda-k} d \lambda\right), \quad \operatorname{Im} k>0,
$$

and

$$
\rho(k)=-\frac{r(-k) t(k)}{t(-k)} .
$$

Using (3.23) and (3.24) we can then calculate the transmission coefficient. We only state the result and refer to [2] for a detailed discussion of the inverse scattering problem for rational reflection coefficients. We have

$$
t(k)=\frac{k(k+\alpha i)}{(k+i)(k+2 i)}, \quad \alpha=\sqrt{5},
$$

and using (3.25),

$$
\rho(k)=\frac{-2(k+\alpha i)}{(k+i)(k+2 i)(k-\alpha i)} .
$$

To determine the potential for $x<0$, we use the Gelfand-Levitan equation for the left scattering data,

$$
R(x, y)+Q(x+y)+\int_{-\infty}^{x} R(x, z) Q(z+y) d z=0 \quad \text { when } \quad x>y
$$

where

$$
Q(x)=\frac{1}{2 \pi} \int_{-\infty}^{+\infty} \rho(k) e^{-i x k} d k .
$$

The residue calculus then gives that

$$
Q(x)=\frac{-4 \alpha}{(\alpha+1)(\alpha+2)} e^{\alpha x}, \quad x<0,
$$

and therefore solving (3.27), we find that the potential is given by

$$
p(x)=\frac{16 \alpha^{2} \mu e^{2 \alpha x}}{\left(\mu-2 e^{2 \alpha x}\right)^{2}}, \quad x<0
$$

where $\mu=(\alpha+1)(\alpha+2)$. The transmission coefficient $t(k)$ admits a meromorphic continuation to the set $\operatorname{Im} k>-\alpha$, and the poles there are the resonances of $p$. From (3.26) we see that there are only two resonances, both situated on the imaginary axis. 
The example above admits a direct generalization which we shall finally describe. When doing this we start with a function $R(x, y)$ in the form

$$
R(x, y)=\left(u(x) e^{\alpha(y-x)} \theta_{-}(x)+(f(x-y)+g(x+y)) \theta_{+}(x)\right) \theta_{+}(x-y) .
$$

Here $\theta_{-}(x)=\theta_{+}(-x)$ and $\alpha$ is a positive number. The functions $f, g$ and $u$ are to be chosen so that $I+R$ will be the intertwining operator $A_{-}$corresponding to a potential $p$, supported by $\overline{\mathbf{R}_{-}}$. We then must have that

$$
\left(\partial_{x}^{2}-\partial_{y}^{2}\right) R(x, y)=p(x) R(x, y)+p(x) \delta(x-y) .
$$

If we require that

$$
f(-y)+g(y)=u(0) e^{\alpha y}, \quad y \leq 0
$$

then

$$
\left(\partial_{x}+\partial_{y}\right) R(x, y)=\left(u^{\prime}(x) e^{\alpha(y-x)} \theta_{-}(x)+2 g^{\prime}(x+y) \theta_{+}(x)\right) \theta_{+}(x-y),
$$

and hence if

$$
2 g^{\prime}(y)=u^{\prime}(0) e^{\alpha y}, \quad y \leq 0
$$

we obtain

$$
\begin{aligned}
\left(\partial_{x}^{2}-\partial_{y}^{2}\right) R(x, y) & =2\left(u^{\prime}(x) \theta_{-}(x)+2 g^{\prime}(2 x) \theta_{+}(x)\right) \delta(x-y) \\
& +\left(u^{\prime \prime}(x)-2 \alpha u^{\prime}(x)\right) e^{\alpha(y-x)} \theta_{-}(x) \theta_{+}(x-y) .
\end{aligned}
$$

If we choose $u$ such that

$$
u^{\prime \prime}(x)-2 \alpha u^{\prime}(x)=2 u(x) u^{\prime}(x), \quad x<0,
$$

and if finally

$$
g^{\prime}(x)=0, \quad x>0,
$$

then it follows that $R$ satisfies (3.30) with $p(x)=2 u^{\prime}(x) \theta_{-}(x)$. Assuming that $u$ is not identically zero, solving (3.33) we find that

$$
u(x)=\frac{2 \alpha e^{2 \alpha x}}{2 \alpha C-e^{2 \alpha x}}, \quad x<0,
$$

for some $C$ with $2 \alpha C>1$, and we now have to choose $f$ and $g$ so that the conditions (3.31), (3.32) and (3.34) are satisfied. Now (3.32) together with (3.34) gives that $g(y)=\left(u^{\prime}(0) / 2 \alpha\right) e^{\alpha y} \theta_{-}(y)+\left(u^{\prime}(0) / 2 \alpha\right) \theta_{+}(y)$, since $g$ must be continuous. Then by (3.31) we get

$$
f(y)=u(0) e^{-\alpha y}-\frac{u^{\prime}(0)}{2 \alpha} e^{-\alpha y}, \quad y>0 .
$$

and we have constructed $R$ such that $I+R$ is the intertwining operator $A_{-}$.

Using (3.29) it is now easy to compute $X_{p}$. Since for $x<0$ we have that

$$
R(x, y)=u(x) e^{\alpha(y-x)} \theta_{+}(x-y),
$$


and $x \leq 0$ in the support of $p$, we get

$$
\int p(z) R(z, z+y) d z=\left(\int_{-\infty}^{0} p(z) u(z) d z\right) e^{\alpha y} \theta_{-}(y)=u^{2}(0) e^{\alpha y} \theta_{-}(y)
$$

since $p(x)=2 u^{\prime}(x)$. Then

$$
X_{p}(y)=\delta^{\prime}(y)-u(0) \delta(y)-\frac{1}{2} \theta_{-}(y) u^{2}(0) e^{\alpha y} .
$$

The conclusion that $\hat{X}_{p}$ has only finitely many zeros is now immediate. In particular, in the special case when $R$ comes from the potential given by (3.28), computing the Fourier transform in (3.35) we recover the expression (3.26).

\subsection{A coupled system of Riccati equations}

The purpose of this section is to present an alternative approach to the study of the location of resonances. It is more direct than before and does not depend on the study of the scattering matrix. Instead we shall work with a system of Riccati equations. Notice that a related approach has been used in [1] when studying stability of the shape resonances.

Working with Riccati equations will allow us to recover the results concerning the purely imaginary poles, and also, we shall derive bounds giving improved estimates on the imaginary part of the poles with sufficiently large real part. A further study of the system of the Riccati equations (3.37) below could perhaps lead to more precise estimates.

We assume first that $p \in L^{1}$ is compactly supported and $\operatorname{supp}(p) \subset[a, b]$. For $k \in \mathbf{C}_{-}$we let $u(x, k)$ be the solution to the problem

$$
H_{p} u=k^{2} u, \quad u(x, k)=e^{-i x k}, \quad x<a .
$$

We want to investigate when

$$
u(x, k)=c e^{i k x} \quad \text { for } \quad x>b .
$$

Consider the function

$$
\varphi(x, k)=\frac{u_{x}^{\prime}(x, k)}{u(x, k)}
$$

which solves the Riccati equation

$$
\varphi^{\prime}=p-k^{2}-\varphi^{2}
$$

in the set where $u \neq 0$. In what follows we write $k=\alpha-i \beta$, where $\beta>0$, and $\alpha$ will be kept fixed. If instead of $\varphi$ we consider

$$
\psi=\varphi+i k
$$


then

$$
\psi^{\prime}=p+2 i k \psi-\psi^{2}, \quad \psi(x)=0, \quad x<a .
$$

For reasons of symmetry we may assume that $\alpha \geq 0$, and we write

$$
\psi=f+i g
$$

This gives us a coupled system of ODE:

$$
\left\{\begin{array}{l}
f^{\prime}=p+2 \beta f-2 \alpha g-f^{2}+g^{2} \\
g^{\prime}=2 \alpha f+2 \beta g-2 f g
\end{array}\right.
$$

and $f(x)=g(x)=0$ for $x<a$. We know that $\alpha-i \beta$ is a resonance precisely when $f(b, \beta)=2 \beta$ and $g(b, \beta)=2 \alpha$. First, we shall examine the situation when $\alpha=0$ and $p \geq 0$. In this case the description of the resonances is given by Proposition 3.4, but it is instructive to recover these results by studying (3.37). Then $g=0$ and we have the equation

$$
f^{\prime}=p+2 \beta f-f^{2} .
$$

Since $f(x)=0$ for $x \leq a$ and $p(x) \geq 0$, it is true that $f(x) \geq 0$ where it is defined. Then

$$
f(x) \leq \int_{a}^{x} e^{2 \beta(x-y)} p(y) d y
$$

and it follows that $f$ exists on the whole interval $[a, b]$. Notice also that Proposition 3.4 gives

$$
\|p\|_{L^{1}}>2 \beta \Rightarrow-i \beta \quad \text { is not a scattering pole. }
$$

Next we shall study the derivative $f_{\beta}^{\prime}$ of $f$ with respect to $\beta$. We have

$$
f_{x \beta}^{\prime \prime}=2 \beta f_{\beta}^{\prime}+2 f-2 f f_{\beta}^{\prime}
$$

and $f_{\beta}^{\prime}(a, \beta)=0$. Since $f \geq 0$, it follows from this equation that $f_{\beta}^{\prime}(x, \beta) \geq 0$. Then

$$
f_{x \beta}^{\prime \prime} \leq 2 \beta f_{\beta}^{\prime}+2 f
$$

and it implies that

$$
f_{\beta}^{\prime}(x, \beta) \leq 2 e^{2 x \beta} \int_{a}^{x} e^{-2 y \beta} f(y, \beta) d y \leq 2 e^{2 b \beta} \int_{a}^{b} e^{-2 y \beta} f(y, \beta) d y, \quad x \leq b .
$$

Now, in view of (3.38),

$$
\begin{aligned}
& \int_{a}^{b} e^{-2 y \beta} f(y, \beta) d y=\int_{a}^{b}(b-y) \partial_{y}\left(e^{-2 y \beta} f(y, \beta)\right) d y \\
= & \int_{a}^{b}(b-y) p(y) e^{-2 \beta y} d y-\int_{a}^{b}(b-y) f^{2}(y) e^{-2 y \beta} d y \leq \int_{a}^{b}(b-y) p(y) e^{-2 \beta y} d y .
\end{aligned}
$$


Define the function

$$
\varphi(p)=\sup \left\{\beta \geq 0, e^{2 b \beta} \int_{a}^{b}(b-y) p(y) e^{-2 \beta y} d y<1\right\} .
$$

Then $\varphi(p) \geq \beta_{0}$, where $e^{2 \beta_{0}(b-a)}(b-a)\|p\|_{L^{1}}=1$. Since $f_{\beta}^{\prime}(b, \beta)<2$ on $\left(0, \beta_{0}\right)$, it follows that the equation $f(b, \beta)=2 \beta$ has at most one solution on this interval. Taking into account (3.39), we summarize the discussion above in the following result, which is just a restatement of Proposition 3.3, combined with (3.39).

Proposition 3.12 Let $0 \leq p \in L^{1}$ be supported by an interval of length $d>0$. Put

$$
\eta(p)=\max \left(\frac{1}{2}\|p\|_{L^{1}}, \frac{1}{2 d} \log \left(\frac{1}{d\|p\|_{L^{1}}}\right)\right) .
$$

Then there can be at most one resonance of the form $-i \beta$, when $\beta \in(0, \eta(p))$.

In the case of poles off the imaginary axis and in the case when the potential has variable sign, the situation becomes more subtle, and it is no longer clear that the coupled system of equations (3.37) has a global solution. To circumvent this difficulty, we view the function $\varphi$, defined in (3.36) as taking values in the complex projective line $\mathbf{C} P^{1}$. If we use $u$ and $u^{\prime}$ as a system of homogeneous coordinates, then the (nonautonomuous) vector field $X$, generating the global flow, is given by

$$
X\left(u: u^{\prime}\right)=p-k^{2}-\left(\frac{u^{\prime}}{u}\right)^{2}
$$

on an open set where $u \neq 0$, and

$$
X\left(u: u^{\prime}\right)=1-\left(p-k^{2}\right)\left(\frac{u}{u^{\prime}}\right)^{2}
$$

where $u^{\prime} \neq 0$. It is now convenient to introduce new homogeneous coordinates $k u-i u^{\prime}$ and $k u+i u^{\prime}$, so that the solution curve starts at the point $(0: 1)$. We may then formulate the condition that $k$ is a pole by saying that the solution curve passes through the point $(1: 0)$ at time $x=b$. A straightforward computation, using, for example, transition functions for the tangent bundle of $\mathbf{C} P^{1}$, gives the expression for the vector field $X$ on an open set, where $k u+i u^{\prime} \neq 0$. We get

$$
X=\frac{p}{2 i k}\left(1+\frac{k u-i u^{\prime}}{k u+i u^{\prime}}\right)^{2}+2 i k \frac{k u-i u^{\prime}}{k u+i u^{\prime}} .
$$

Therefore, the function

$$
f(x, k)=\frac{k u-i u^{\prime}}{k u+i u^{\prime}}
$$


vanishes for $x<a$, and solves the differential equation

$$
f_{x}^{\prime}=\frac{p}{2 i k}(1+f)^{2}+2 i k f .
$$

Our aim now is to estimate the lifespan of the solution. In particular, if $f$ exists on the entire interval $(-\infty, b]$, then $k$ is not a resonance. To that end, we shall derive a differential inequality for $|f|$. A computation shows that

$$
\frac{d}{d x}|f|^{2}=4 \beta|f|^{2}+\frac{2 p \beta|f|^{2}}{|k|^{2}}+\operatorname{Re}\left(\frac{p \bar{f}}{i k}\right)+p|f|^{2} \operatorname{Re}\left(\frac{f}{i k}\right),
$$

and therefore, for $|f(x)| \neq 0$, we have

$$
\frac{d}{d x}|f| \leq 2 \beta|f|+\frac{|p||f|}{|k|}+\frac{|p|}{2|k|}+\frac{|p||f|^{2}}{2|k|} .
$$

If $g$ solves

$$
g^{\prime}(x)=2 \beta g(x)+\frac{|p(x)| g(x)}{|k|}+\frac{|p(x)|}{2|k|}+\frac{|p(x)| g^{2}(x)}{2|k|}
$$

and $g$ vanishes for $x=a$, then, by comparison, we have that $0 \leq|f| \leq g$, where $g$ is defined. To estimate the lifespan of $g$, we apply the following lemma.

Lemma 3.13 Consider a nonlinear differential equation

$$
\left\{\begin{array}{l}
h^{\prime}(t)=a(t) h^{2}(t)+b(t) \\
h(0)=0
\end{array}\right.
$$

where $a$ and $b$ are nonnegative locally integrable functions. If

$$
\left(\int_{0}^{T} a(t) d t\right)\left(\int_{0}^{T} b(t) d t\right)<1
$$

then the solution $h(t)$ exists on $[0, T]$.

Proof: This follows from Lemma 1.3.3 in [9].

To apply the lemma, we just write

$$
g(x)=h(x) \exp (2 \beta x+(1 /|k|) P(x)),
$$

where

$$
P(x)=\int_{-\infty}^{x}|p(y)| d y
$$

so that $h$ solves

$$
h^{\prime}(x)=\frac{|p(x)|}{2|k|} \exp (2 \beta x+(1 /|k|) P(x)) h^{2}(x)+\frac{|p(x)|}{2|k|} \exp (-2 \beta x-(1 /|k|) P(x)) \text {. }
$$


Using that the product of the integrals of the coefficients in (3.41) is less than or equal to

$$
\frac{1}{4|k|^{2}} \exp \left(\|p\|_{L^{1}} /|k|\right) \iint e^{2 \beta|x-y|}|p(x) p(y)| d x d y,
$$

we arrive at the following theorem.

Theorem 3.14 Let $p$ be super-exponentially decaying. Then if $k=\alpha-i \beta, \beta>0$, is a scattering pole of $p$, we have

$$
\frac{1}{4|k|^{2}} \exp \left(\|p\|_{L^{1}} /|k|\right) \iint e^{2 \beta|x-y|}|p(x) p(y)| d x d y \geq 1 .
$$

Proof: We have already observed that if $p$ is compactly supported, the assertion is a direct application of Lemma 3.13 to (3.41). In the general case, we choose a sequence $p_{j} \in L^{1}$ of compactly supported functions such that $\left|p_{j}\right| \leq|p|$ and $p_{j} \rightarrow p$ almost everywhere. Then it follows as in Theorem 3.6 that $\hat{X}_{p_{j}} \rightarrow \hat{X}_{p}$ locally uniformly. An application of Hurwitz's theorem shows then that $k$ is a pole of $p$ if and only if $k=\lim _{j \rightarrow \infty} k_{j}$, where $k_{j}$ is a pole of $p_{j}$. Applying (3.42) to each $p_{j}$ and letting $j \rightarrow \infty$ gives the theorem.

We can remark here that when $p$ is compactly supported, then Theorem 3.14 gives a direct proof of the logarithmic bound for the imaginary part of the poles in this case.

Remark. We finally notice that a result, similar to Theorem 3.14 can be obtained if one uses the characterization of the resonances as poles of the meromorphic continuation of the weighted resolvent of the Schrödinger operator. In fact, it is essentially well known (see, for example, [5]) and follows from the resolvent equation combined with the analytic Fredholm theory, that, for a super-exponentially decaying potential, the weighted resolvent

$$
R_{p}(k)=p^{1 / 2} R(k)|p|^{1 / 2}
$$

admits a meromorphic continuation to the lower half-plane. Here $p^{1 / 2}=\operatorname{sign}(p)|p|^{1 / 2}$ and

$$
R(k)=\left(H_{p}-k^{2}\right)^{-1}, \quad \operatorname{Im} k>0, \quad k^{2} \notin \sigma\left(H_{p}\right) .
$$

Moreover, the poles of the continuation are precisely the points $k$ such that the weighted free resolvent

$$
R_{0, p}(k)=p^{1 / 2} R_{0}(k)|p|^{1 / 2}
$$

has -1 as an eigenvalue. Since

$$
R_{0}(k)(x, y)=i \frac{e^{i k|x-y|}}{2 k}
$$

it follows that (3.43) is an analytic family of Hilbert-Schmidt operators for $k \neq 0$. Therefore if $k$ is such that the Hilbert-Schmidt norm of $R_{0, p}(k)$ is less than one, then 
$k$ is not a pole. This leads to an estimate, similar to (3.42).

Acknowledgements. I am deeply grateful to Professor Anders Melin for his invaluable advice and encouragement during the preparation of this paper. I am also grateful to Professor Lars Hörmander for communicating the new and improved proof of Proposition 3.9 .

\section{References}

[1] Ashbaugh, M., Sundberg, C.: An improved stability result for resonances. Trans. Amer. Math. Soc. 281, 347-360 (1984)

[2] Calogero, F., Degasperis, F.: Spectral transform and solitons. Amsterdam: NorthHolland, 1982

[3] Deift, P., Trubowitz, E.: Inverse scattering on the line. Comm. Pure Appl. Math. 32, 121-251 (1979)

[4] Fernandez, C., Lavine, R.: Lower bounds for resonance widths in potential and obstacle scattering. Commun. Math. Phys. 128, 263-284 (1990)

[5] Froese, R.: Asymptotic distribution of resonances in one dimension. J. Diff. Eq. 137, 251-272 (1997)

[6] Froese, R.: Upper bounds for the resonance counting function in odd dimensions. Can. J. Math. 50(3), 538-546 (1998)

[7] Harrell II, E.M.: General lower bounds for resonances in one dimension. Commun. Math. Phys. 86, 221-225 (1982)

[8] Hörmander, L.: The Analysis of Linear Partial Differential Operators I. BerlinNew York: Springer-Verlag, 1983

[9] Hörmander, L.: Lectures on nonlinear hyperbolic differential equations. Berlin: Springer-Verlag, 1997

[10] Hörmander, L.: Notions of Convexity. Boston: Birkhäuser, 1994

[11] Hörmander, L., Sigurdsson, R.: Growth properties of plurisubharmonic functions related to Fourier-Laplace transforms. Preprint, Department of Mathematics, Lund University, 1993

[12] Lax, P.D., Phillips, R.S.: A logarithmic bound on the location of the poles of the scattering matrix. Arch. Rat. Mech. 40, 268-280 (1971) 
[13] Marchenko, V. A.: Sturm-Liouville operators and applications. Basel: Birkhäuser Verlag 1986

[14] Melin, A.: Operator methods for inverse scattering on the real line. Comm. P.D.E. 10, 677-786 (1985)

[15] Sá Barreto, A., Zworski, M.: Existence of resonances in potential scattering. Comm. Pure Appl. Math. 173, 1271-1280 (1996)

[16] Titchmarsh, E. C.: The theory of functions. Oxford: Oxford Univerisity Press 1968.

[17] Zworski, M.: Distribution of poles for scattering on the real line. J. Funct. Anal. 73, 277-296 (1987)

[18] Zworski, M.: Counting scattering poles. In: Ikawa, M. (ed.) Spectral and Scattering Theory. pp. 301-331. New York: Marcel Dekker 1994 